\title{
SIMILARITY SOLUTIONS TO BURGERS' EQUATION IN TERMS OF SPECIAL FUNCTIONS OF MATHEMATICAL PHYSICS
}

\author{
Turgut Öziș ${ }^{\dagger}$
}

Department of Mathematics, Ege University, Bornova, İzmir, Turkey

İSMAİL ASLAN

Department of Mathematics, Izmir Institute of Technology, Urla, İzmir, Turkey

(Received March 28, 2017; accepted June 26, 2017)

In this paper, the Lie group method is used to investigate some closed form solutions of famous Burgers' equation. A detailed and complete symmetry analysis is performed. By similarity transformations, the equation is reduced to ordinary differential equations whose general solutions are written in terms of the error function, Kummer's confluent hypergeometric function $\Phi(a, b ; x)$ and Bessel functions $J_{p}$, showing the strong connection between the best mathematical modelling equations and the special functions of mathematical physics.

DOI:10.5506/APhysPolB.48.1349

\section{Introduction}

Most of phenomena in the nature are non-linear and modelled by nonlinear equations. One of the most celebrated quasi-linear parabolic partial differential equations, which governs the competition between convection and diffusion, is Burgers' equation

$$
u_{t}+u u_{x}=\nu u_{x x}, \quad \nu>0,
$$

where $t$ and $x$ represent time and distance in $x$-direction, respectively, and $\nu$ is the kinematic viscosity parameter related to the Reynolds number $\operatorname{Re}(=$ $1 / \nu)$. The term $u u_{x}$ represents a non-linear first order convection or a transport term and the term $\nu u_{x x}$ represents a second order (Fickian) diffusion

\footnotetext{
$\dagger$ Corresponding author: turgut.ozis@ege.edu.tr
} 
term. On the one hand, the non-linear convection term has a shocking up effect on an initial waveform, while the diffusion term attempts to smear out the solution. Equation (1) is a balance between these two effects. It is often taken as the analog equation of compressible, viscous fluid flow; in that case, the diffusion term is interpreted as a model viscosity term, which is also a dissipative term that tends to smear out signals [1]. Hence, $u(x, t)$ is the velocity for space $x$ and time $t, \nu>0$ is the kinematics viscosity parameter related to the Reynolds number $\operatorname{Re}(=1 / \nu)$. Burgers' equation is also the lowest order approximation for the one-dimensional propagation of weak shock waves in a fluid. Further, Burgers' equation can be considered as a simplified form of the Navier-Stokes equation, namely, it is a model for statistical theory of the turbulence which can be thought of as a one-dimensional scalar analogous of the Navier-Stokes equations of fluid dynamics $[2,3]$.

This model has been also studied for the following reasons:

I. Its analytical solution exists for some set of initial conditions so that a numerical comparison can be made;

II. Its shock wave behaviour when Reynolds number, Re, is large.

Historically, the steady state solutions of Burgers' equation were first introduced by Bateman [4] in 1915 in modelling the motion of a fluid with small viscosity $\nu$, and later analysed in detail by Burgers [2] in 1948 that this equation can be used to confine some of the futures of turbulent fluid in a channel originated by the relation of opposite effects of convection and diffusion. The complete work of J.M. Burgers may be found in his famous book [3]. Burgers' equation can also be used for description of the structure of the shock waves in a viscous medium, sound waves in a viscous medium, waves in fluid-filled viscous elastic tubes, magnetohydrodynamic waves in a medium with finite electrical conductivity and traffic flow [5].

If $\nu=0$ in equation (1), the equation is called inviscid Burgers' equation and describes gas dynamics. By setting $u=v_{x}$ and integrating once with respect to $x$, and discarding an arbitrary function of $t$, equation (1) becomes $v_{t}+\left(\frac{1}{2}\right) v_{x^{2}}=\nu v_{x x}$, an equation which has some significance in the burning of gas. Further, if we set $v=-2 \nu \log (w)$, it transforms the equation to $w_{t}=\nu w_{x x}$ to the one-dimensional diffusion equation [6].

It is noteworthy that Burgers' equation may be solved exactly using the transformation $u=-2 \nu \phi_{x} / \phi$, discovered independently by Hopf [7] and Cole [8] in early 1950s. They showed that Burgers' equation may be transformed to the linear heat equation $\phi_{t}=\phi_{x x}$ by a non-linear transformation the so-called Cole-Hopf transformation, and it was a landmark in the modern time of non-linear partial differential equations. 
It was also shown by Cole [8] that equation (1) is derivable from NavierStokes equation in the limit of a weak shock layer.

It is worth recalling that equation (1) is of a parabolic type, while (1) with $\nu=0$ is hyperbolic, hence, the properties of the solution of the parabolic equation are extensively different from those of the hyperbolic type equation. Equation (1) is a primary evolution equation that emerges in a number of unrelated applications where both viscosity and non-linearity are equally important.

Therefore, to understand a range of physical phenomenon, for example, Lighthill [9] showed that Burgers' equation is an appropriate model to investigate the weak planar wave incident. Blackstock [10], Walsh [11], Benton and Platzman [12], Crighton and Scott [13], Parker [14], Rodin [15], Larson [16], and Lardner and Arya [17] examined initial boundary-value problems for the Burgers' equation and its solutions along with their physical significance. Benton and Platzman [12] have catalogued all available solutions of Burgers' equation and have illustrated the physically interesting ones. Various generalizations of Burgers' equation have been given by a number of researchers including Case and Chiu [18], Murray [19-21], Rosenblatt [22], Penel and Brauner [23], and Crighton [24]. Kriess and Lorenz [25] investigated the existence and uniqueness of solutions of boundary value problems associated with the Burgers' equation. For more compact introduction to the subject, readers are asked to refer to the outstanding books by Debnath [5] and Kevorkian [26] and the references therein.

A great deal of research has been invested on Burgers' equation to seek exact and explicit solutions by using distinct approaches. In the past few decades, some researchers have made many attempts and today there exist various methods which are powerful and appropriate to solve such equations. Among them are: the tanh-coth method [27], the modified extended tanh function method [28], exp function method [29], Adomian's decomposition method [30, 31], inverse scattering method [32], Hirota's bilinear method [33], Painleve Ansatz [34], Lie group method [35-41], etc.

At the same time, however, since the analytic solutions are available for some limiting cases, i.e., some set of initial functions, it is natural to consider Burgers' equation for testing the performance of a numerical solver. Thus, many interesting papers on the numerical solution of Burgers' equation based on finite difference, finite element, boundary element and spectral methods, etc. exist in the literature. For example, Miller [36] has investigated and compared predictor-corrector method with the explicit method and exact solution for an initial-boundary value problem given by Burgers' equation. It is not a purpose of this paper to cite all existing papers for solving Burgers' equation with numerical solver, but to point out some of them that are worthwhile [42-59]. 
The main purpose of this paper is to show systematically that the application of Lie groups of infinitesimal transformations to Burgers' equation in the extended space of both independent and dependent variables enables one to obtain similarity reductions and to draw attention to some physically interesting analytic solutions written in terms of special functions of mathematical physics such as the error function, Kummer's confluent hypergeometric function and Bessel function. This will prove the existence of a strong connection among very important (currently existing) best modelling equations and functions describing real world phenomena.

The rest of this paper is organized as follows: In Section 2, we consider Burgers' equation with $\nu=1$ and provide the general form of the infinitesimal Lie point transformations which will be the full group leaving the equation invariant and demonstrate the finite form of each infinitesimal subgroups. In Section 3, symmetry reduction is used to obtain particular solutions. The last section makes some concluding remarks.

\section{Lie symmetry analysis of Eq. (1) with $\nu=1$}

The symmetry analysis of differential equations using group theory goes back to M.S. Lie. Lie point transformations are the ones which are mostly used. These transformations which leave the equation invariant act on the space of the dependent and independent variables. Revealing the symmetries of the equation by Lie's method perhaps enables us to obtain new solutions directly or from the known ones or via similarity reductions. The method for finding the Lie point symmetries is well-known and has been wellestablished in the last few decades, see, for example, Bluman and Anco [60], Ibragimov [61, 62], Olver [35], Ovsiannikov [63], and Leach and Govinde [64]. Symmetries, which are transformations that reduce the number of independent variables of a partial differential equation can be applied to derive similarity solutions.

In this section, as a practical matter, we consider Burgers' equation (1) with the kinematic viscosity $\nu=1$

$$
u_{t}+u u_{x}=u_{x x}
$$

To begin with, we would like to mention that it is not an easy task to guess a Lie point group in finite form which leaves Eq. (2) invariant. We shall achieve the reduction of Eq. (2) to an ordinary differential equation by means of various similarity reductions. This reduced equation is of fewer variables and generally easier to solve. The first step of similarity reductions is to determine all infinitesimal generators of symmetry group of the given differential equation. In doing so, let us assume that the infinitesimal form 
of a one-parameter $(\varepsilon)$ continuous transformations group (Lie group) acting on $(x, t, u)$ — space admitted by Eq. (2) has the form of

$$
\begin{aligned}
& \bar{x}=x+\varepsilon \xi_{1}(x, t, u)+O\left(\varepsilon^{2}\right), \\
& \bar{t}=t+\varepsilon \xi_{2}(x, t, u)+O\left(\varepsilon^{2}\right), \\
& \bar{u}=u+\varepsilon \eta(x, t, u)+O\left(\varepsilon^{2}\right)
\end{aligned}
$$

up to the first order of $\varepsilon$. The symmetry algebra is realized by the set of vector fields of the form of $L=\xi_{1} \partial_{x}+\xi_{2} \partial_{t}+\eta \partial_{u}$, where $\partial_{x} \equiv \partial / \partial_{x}$, etc. The infinitesimals $\xi_{1}(x, t, u), \xi_{2}(x, t, u)$ and $\eta(x, t, u)$ are to be determined from the invariance condition. Using the second prolongation $L^{(2)}=\xi_{1} \partial_{x}+$ $\xi_{2} \partial_{t}+\eta \partial_{u}+\pi^{x} \partial_{u_{x}}+\pi^{t} \partial_{u_{t}}+\pi^{x x} \partial_{u_{x x}}$ and keeping only the necessary terms, annihilates Eq. (2) on its solution set. The extended infinitesimals $\pi^{x}, \pi^{t}$ and $\pi^{x x}$ are derived, by the standard procedure [8, 9], as

$$
\begin{aligned}
& \pi^{x}\left(x, t, u, u_{x}, u_{t}\right)=\eta_{x}+\eta_{u} u_{x}-\left(\xi_{1}\right)_{x} u_{x}-\left(\xi_{1}\right)_{u} u_{x}^{2}-\left(\xi_{2}\right)_{x} u_{t}-\left(\xi_{2}\right)_{u} u_{x} u_{t}, \\
& \pi^{t}\left(x, t, u, u_{x}, u_{t}\right)=\eta_{t}+\eta_{u} u_{t}-\left(\xi_{1}\right)_{t} u_{x}-\left(\xi_{2}\right)_{u} u_{t}^{2}-\left(\xi_{2}\right)_{t} u_{t}-\left(\xi_{1}\right)_{u} u_{x} u_{t}, \\
& \pi^{x x}\left(x, t, u, u_{x}, u_{t}, u_{x x}, u_{x t}, u_{t t}\right)=\eta_{x x}+\left(2 \eta_{x u}-\left(\xi_{1}\right)_{x x}\right) u_{x}-\left(\xi_{2}\right)_{x x} u_{t} \\
& +\left\{\eta_{u u}-2\left(\xi_{1}\right)_{x u}\right\} u_{x}^{2}-2\left(\xi_{2}\right)_{x u} u_{t} u_{x}-\left(\xi_{1}\right)_{u u} u_{x}^{3}-\left(\xi_{2}\right)_{u u} u_{t} u_{x}^{2} \\
& -2\left\{\left(\xi_{2}\right)_{x}+\left(\xi_{2}\right)_{u} u_{x}\right\} u_{x t}+\left\{\left(\eta_{u}-2\left(\xi_{1}\right)_{x}\right)-3\left(\xi_{1}\right)_{u} u_{x}-\left(\xi_{2}\right)_{u} u_{t}\right\} u_{x x} .
\end{aligned}
$$

Taking $H=u_{t}+u u_{x}-u_{x x}$, the determining system of Eq. (2) arises from the invariance condition $\left.L^{(2)} H\right|_{H=0}=0$. This results in the equation $\eta u_{x}+$ $\pi^{x} u+\pi^{t}-\pi^{x x}=0$ and thus the equation

$$
\begin{aligned}
& \left(\eta_{t}-\eta_{x x}\right)+\eta_{x} u+\left\{\eta-\left(\xi_{1}\right)_{t}-2 \eta_{x u}+\left(\xi_{1}\right)_{x x}\right\} u_{x}+\left(\xi_{1}\right)_{x} u u_{x}-\left(\xi_{2}\right)_{x} u u_{t} \\
& +\left\{\left(\xi_{1}\right)_{u}+\left(\xi_{2}\right)_{x u}\right\} u_{x} u_{t}+2\left(\xi_{1}\right)_{u} u u_{x}^{2}+\left\{2\left(\xi_{1}\right)_{x u}-\eta_{u u}\right\} u_{x}^{2}+\left(\xi_{1}\right)_{u u} u_{x}^{3} \\
& +\left(\xi_{2}\right)_{u u} u_{t} u_{x}^{2}+2\left(\xi_{2}\right)_{x} u_{x t}+2\left(\xi_{2}\right)_{u} u_{x} u_{x t}=0 .
\end{aligned}
$$

Equating the coefficients of the derivatives $u^{0}, u, u_{x}, u u_{x}, u u_{x}^{2}, u u_{t}, u_{x} u_{t}, u_{x}^{2}$, $u_{x}^{3}, u_{t} u_{x}^{2}, u_{x t}, u_{x} u_{x t}$ to zero gives the determining system

$$
\begin{aligned}
& \eta-\left(\xi_{1}\right)_{t}-2 \eta_{x t t}+\left(\xi_{1}\right)_{x x}=0, \quad\left(\xi_{1}\right)_{x}=0, \quad\left(\xi_{1}\right)_{u}+\left(\xi_{2}\right)_{x u}=0, \\
& \eta_{x}=0, \quad \eta_{t}-\eta_{x x}=0, \quad\left(\xi_{1}\right)_{u}=0, \quad-\eta_{u u}+2\left(\xi_{1}\right)_{x u}=0, \\
& \left(\xi_{2}\right)_{u}=0, \quad\left(\xi_{2}\right)_{u u}=0, \quad\left(\xi_{1}\right)_{u u}=0, \quad\left(\xi_{2}\right)_{x}=0 \text {, } \\
& -\left(\xi_{2}\right)_{t}+2\left(\xi_{1}\right)_{x}+\left(\xi_{2}\right)_{x x}=0, \quad\left(\xi_{2}\right)_{x}=0 \text {. }
\end{aligned}
$$

After some calculations, the arising determining equations lead to the following infinitesimals:

$$
\begin{aligned}
\xi_{1}(x, t, u) & =a_{1}-a_{2} x+a_{3} t-a_{4} x t, \\
\xi_{2}(x, t, u) & =a_{5}-2 a_{2} t-a_{4} t^{2}, \\
\eta(x, t, u) & =a_{3}+a_{2} u+a_{4}(t u-x),
\end{aligned}
$$


where $a_{1}, a_{2}, a_{3}, a_{4}$ and $a_{5}$ are constants. The associated vector fields for the one-parameter Lie group of infinitesimal transformations are $X_{1}, X_{2}, \ldots X_{5}$, given by

$$
\begin{aligned}
& X_{1}=\partial_{x}, \quad X_{2}=\partial_{t}, \quad X_{3}=t \partial_{x}+\partial_{u}, \\
& X_{4}=-x \partial_{x}-2 t \partial_{t}+u \partial_{u}, \quad X_{5}=-t x \partial_{x}-t^{2} \partial_{t}+(t u-x) \partial_{u} .
\end{aligned}
$$

By solving Lie equations, the following list presents the finite one-parameter groups:

$$
\begin{aligned}
\bar{x} & =f(x, t, u, \varepsilon), \\
\bar{t} & =g(x, t, u, \varepsilon), \\
\bar{u} & =h(x, t, u, \varepsilon)
\end{aligned}
$$

corresponding to operators $X_{i}, 1 \leq i \leq 5$. In each case, $\varepsilon$ is a continuous group parameter. The entries give the transformed point $\exp \left(\varepsilon X_{i}\right)(x, t, u)=$ $(\bar{u}, \bar{t}, \bar{u})$ :

$$
\begin{aligned}
\exp \left(\varepsilon X_{1}\right):(x, t, u) & \mapsto(x+\varepsilon, t, u) \\
\exp \left(\varepsilon X_{2}\right):(x, t, u) & \mapsto(x, t+\varepsilon, u) \\
\exp \left(\varepsilon X_{3}\right):(x, t, u) & \mapsto(x+\varepsilon t, t, u,+\varepsilon) \\
\exp \left(\varepsilon X_{4}\right):(x, t, u) & \mapsto\left(e^{-\varepsilon} x, e^{-2 \varepsilon} t, e^{\varepsilon} u\right), \\
\exp \left(\varepsilon X_{5}\right):(x, t, u) & \mapsto\left(\frac{x}{1+\varepsilon t}, \frac{t}{1+\varepsilon t}, u+(t u-x) \varepsilon\right) .
\end{aligned}
$$

The transformations corresponding to $X_{1}, X_{2}$ and $X_{3}$ represent translation, $X_{4}$ represents scaling invariance and $X_{5}$ projective transformation. $X_{5}$ is a local group of transformations. It is worthy to mention that appearance of $X_{1}, \ldots, X_{5}$ transformations is not obvious from the point of view of fundamental physical transformation. Nice feature of those transformations is that they lead to group invariants (for example, by considering finite transformations of the group and by eliminating the group parameter). Hence, the projective transformation, $X_{5}$, has the invariant $x / t$ which is invariant of one-parameter group considered. The generalization of this idea to multiparameter groups acting in a space with a large number of variables was introduced by Oliver and co-workers [65-67].

Symmetries allow one to find new solutions starting from any known solution as well as to construct invariant solutions. Since each group is a symmetry group, if $\phi=\Phi(x, t)$ is a solution of Eq. (2), so are the functions 


$$
\begin{aligned}
\phi_{1} & =\Phi(x-\varepsilon, t), \\
\phi_{2} & =\Phi(x, t-\varepsilon), \\
\phi_{3} & =\Phi(x-\varepsilon t, t)+\varepsilon, \\
\phi_{4} & =e^{\varepsilon} \Phi\left(e^{\varepsilon} x, e^{2 \varepsilon} t\right), \\
\phi_{5} & =\Phi\left(\frac{x}{1-\varepsilon t}, \frac{t}{1-\varepsilon t}\right),
\end{aligned}
$$

where $\varepsilon$ is any real number.

\section{Group-invariant solutions of Eq. (2) by similarity reductions}

For construction of closed form solutions, the Lie group method supplies two fundamental approaches which are the construction of invariant solutions and group transformations of known solutions. If a group transformation maps a solution to itself, we obtain a group invariant solution reducing the number of independent variables of the equation in question. By considering an invariance under symmetry groups with two or more parameters, further reductions can be done. The second approach is based on the fact that a Lie symmetry group transforms any solution of the equation in question into another solution of the same equation. As a result, any known solution is a source of a multi-parameter class of new solutions if the differential equation under consideration admits a multi-parameter Lie symmetry group.

Let us now use the subgroup classification derived in Section 2 to reduce Eq. (2) to ordinary differential equations. Each sub-algebra will provide a different ordinary differential equation. The solutions of the ordinary differential equations will be invariant under the subgroup used in the reduction. Invariant solutions can be expressed via invariants of the subgroup.

Case (1): $X_{1}=\frac{\partial}{\partial x}$.

In this case, similarity variable and similarity transformation are found, respectively, as $\eta=t$ and $u=f(\eta)$ by solving the characteristic system $\frac{\mathrm{d} x}{1}=\frac{\mathrm{d} t}{0}=\frac{\mathrm{d} u}{0}$ which arises from the invariant surface condition $\bar{u}=u(\bar{x}, \bar{t})$. The reduced equation is $f^{\prime}(\eta)=0$ and this implies the steady-state solutions of Eq. (2) as $u(x, t)=C$, where $C$ is an arbitrary constant.

Case (2): $X_{2}=\frac{\partial}{\partial t}$.

By a similar discussion, from the characteristic system $\frac{\mathrm{d} x}{0}=\frac{\mathrm{d} t}{1}=\frac{\mathrm{d} u}{0}$, the invariants are obtained as $\eta=x, u=f(\eta)$ and thus Eq. (2) leads to reduced equation $f^{\prime \prime}=f^{\prime} f$ which creates shock soliton solutions of Eq. (2) as $u(x, t)=\sqrt{2 C_{1}} \tan \left[\left(x+C_{2}\right) \sqrt{2 C_{1}} / 2\right]$, where $C_{1}>0$ and $C_{2}$ are arbitrary constants. 
Case (3): $X_{3}=t \frac{\partial}{\partial x}+\frac{\partial}{\partial u}$.

From the characteristic system $\frac{\mathrm{d} x}{t}=\frac{\mathrm{d} t}{0}=\frac{\mathrm{d} u}{1}$, the similarity variable and similarity transformation are, respectively, $\eta=t$ and $u=(x / t)+f(\eta)$, and the reduced differential equation is $\eta f^{\prime}+f=0$ which constructs the solution of Eq. (2) as $u(x, t)=(x+C) / t$, where $C$ is an arbitrary constant.

Case (4): $X_{4}=-x \frac{\partial}{\partial x}-2 t \frac{\partial}{\partial t}+u \frac{\partial}{\partial u}$.

By solving the characteristic system $\frac{\mathrm{d} x}{-x}=\frac{\mathrm{d} t}{-2 t}=\frac{\mathrm{d} u}{u}$, we end up with the invariants $\eta=x / \sqrt{t}$ (Boltzmann transformation), $u=(1 / \sqrt{t}) f(\eta)$, and the reduced form of Eq. (2) is

$$
f^{\prime \prime}+\left(\frac{\eta}{2}-f\right) f^{\prime}+\frac{1}{2} f=0
$$

which is a non-autonomous, non-linear ordinary differential equation.

Integration of Eq. (3) is not an easy task. As a first approach, we can separate the non-linear term to the right-hand side and write Eq. (3) as an inhomogeneous equation

$$
f^{\prime \prime}+\frac{\eta}{2} f^{\prime}+\frac{1}{2} f=f f^{\prime}
$$

Two linearly-independent solutions corresponding to the homogeneous equation of (4) are $f_{1}(\eta)=e^{-\eta^{2} / 4} \int^{\eta} e^{s^{2} / 4} \mathrm{~d} s$ and $f_{2}(\eta)=e^{-\eta^{2} / 4}$. The Wronskian is $W\left(f_{1}, f_{2}\right)=\frac{-1}{e^{\eta^{2} / 4}}$. Applying the variation of parameters method to Eq. (4) leads to the particular solution $f_{p}(\eta)=\frac{1}{2} e^{-\eta^{2} / 4} \int^{\eta} f^{2}(s) e^{s^{2} / 4} \mathrm{~d} s$ and thus the general solution of Eq. (4) is

$$
f(\eta)=C_{1} e^{-\eta^{2} / 4} \int^{\eta} e^{s^{2} / 4} \mathrm{~d} s+C_{2} e^{-\eta^{2} / 4}+\frac{1}{2} e^{-\eta^{2} / 4} \int^{\eta} f^{2}(s) e^{s^{2} / 4} \mathrm{~d} s
$$

which is an integral equation. We have not yet been able to solve Eq. (3). Another approach is the realization that Eq. (3) can be written as

$$
f^{\prime \prime}+\left(\frac{\eta}{2} f\right)^{\prime}-\frac{1}{2}\left(f^{2}\right)^{\prime}=0
$$

and the integration of Eq. (5) leads straight to the Riccati equation

$$
f^{\prime}+\frac{\eta}{2} f-\frac{1}{2} f^{2}=k
$$

In most cases, finding the solutions of equations of Riccati-type requires a particular solution to be known which is not easy to do so. However, we can 
take advantage of two cases here. For the case of $k=0$, Eq. (6) reduces to a Bernoulli-type equation with the solution

$$
f(\eta)=\frac{-2 e^{-\eta^{2} / 4}}{C+\sqrt{\pi} \operatorname{erf}\left(\frac{\eta}{2}\right)}
$$

resulting in a large family of solutions of Eq. (2) as

$$
u(x, t)=\frac{1}{\sqrt{t}}\left\{\frac{-2 e^{-(x+t)^{2} / 4 t}}{C+\sqrt{\pi} \operatorname{erf}\left(\frac{x+t}{2 \sqrt{t}}\right)}\right\}-1,
$$

where $\operatorname{erf}(z)=\frac{2}{\sqrt{\pi}} \int_{0}^{z} e^{-\theta^{2}} \mathrm{~d} \theta$ is the well-known error function. On the other hand, for the case of $k=1$, it can be observed that a particular solution of Eq. (6) is $f=\eta$. This gives the solution $u(x, t)=x / t$ of Eq. (2) and the substitution $f=\eta+\frac{1}{g}$ in (6) leads to a large family of solutions of the form of

$$
u(x, t)=\frac{1}{\sqrt{t}}\left\{\frac{x+t}{\sqrt{t}}-\frac{2 e^{(x+t)^{2} / 4 t}}{C+\sqrt{\pi} \operatorname{erfi}\left(\frac{x+t}{2 \sqrt{t}}\right)}\right\}-1,
$$

where $\operatorname{erfi}(z)=\operatorname{erf}(i z) / i$ and $i^{2}=-1$.

Now, let us take $k \neq 0,1$ in Eq. (6) and attempt to solve it by a Lie group of infinitesimal transformations. Also, make the exchange $f \leftrightarrow y, \eta \leftrightarrow x$ in Eq. (6) for practical reasons. In order to investigate the infinitesimal transformation

$$
\begin{aligned}
& \bar{x}=x+\varepsilon \xi(x, y)+O\left(\varepsilon^{2}\right), \\
& \bar{y}=y+\varepsilon \eta(x, y)+O\left(\varepsilon^{2}\right)
\end{aligned}
$$

admitted by Eq. (6), we need to find the infinitesimals $\xi$ and $\eta$ from the determining equation

$$
\frac{-y}{2} \xi+\left(y-\frac{x}{2}\right) \eta=\eta_{x}+\left(\eta_{y}-\xi_{x}\right)\left(\frac{1}{2} y^{2}-\frac{x}{2} y+k\right)-\xi_{y}\left(\frac{1}{2} y^{2}-\frac{x}{2} y+k\right)^{2}
$$

which is a linear first-order partial differential equation in $\xi$ and $\eta$. Assuming the function $\xi$ is known, to find the function $\eta$, we need to solve the characteristic system

$$
\begin{aligned}
\frac{\mathrm{d} x}{1} & =\frac{\mathrm{d} y}{\frac{1}{2} y^{2}-\frac{x y}{2}+k} \\
& =\frac{\mathrm{d} \eta}{\xi_{x}\left(\frac{1}{2} y^{2}-\frac{x y}{2}+k\right)+\xi_{y}\left(\frac{1}{2} y^{2}-\frac{x y}{2}+k\right)^{2}-\frac{y}{2} \xi+\left(y-\frac{x}{2}\right) \eta} .
\end{aligned}
$$


However, the first two equations in (8) take us back to the original Eq. (6), so nothing has been achieved. On the other hand, if we apply the transformation

$$
\eta=\xi\left(\frac{1}{2} y^{2}-\frac{x}{2} y+k\right)+\theta(x, y)
$$

suggested by Ovsiannikov [63] for solving determining equations for firstorder ordinary differential equations, to the determining equation (7), it becomes

$$
\theta_{x}+\left(\frac{1}{2} y^{2}-\frac{x}{2} y+k\right) \theta_{y}=\left(y-\frac{x}{2}\right) \theta
$$

whose corresponding characteristic system is

$$
\frac{\mathrm{d} x}{1}=\frac{\mathrm{d} y}{\frac{1}{2} y^{2}-\frac{x}{2} y+k}=\frac{\mathrm{d} \theta}{y-\frac{x}{2}} .
$$

Again, the first two equations in (9) take us back to the original Eq. (6). We have not succeeded in solving (6) completely nor simplifying it any further.

To simplify the form of Eq. (6), as an alternative last approach, we now begin to use a series of transformations. Consulting Zaitsev and Polyanin [68], there is an evidence of possibility that Riccati equation (6) can be converted to a second-order linear differential equation

$$
g^{\prime \prime}+\frac{\eta}{2} g^{\prime}+\frac{k}{2} g=0
$$

by means of the transformation $f=-2 g^{\prime} / g, g=g(\eta)$. Using the Liouville transformation, $g=h \exp \left(-\eta^{2} / 8\right), h=h(\eta)$, one arrives at the so-called canonical (normal form) of Eq. (10) as

$$
h^{\prime \prime}-\left(\frac{\eta^{2}}{16}+\frac{1-2 k}{4}\right) h=0 .
$$

Since the coefficient of the unknown function $h$ in Eq. (11) is a quadratic in $\eta$, we can convert this equation into the confluent hypergeometric equation

$$
z \frac{\mathrm{d}^{2} w}{\mathrm{~d} z^{2}}+\left(\frac{1}{2}-z\right) \frac{\mathrm{d} w}{\mathrm{~d} z}-\frac{1}{2}(1-k) w=0
$$

via the transformation $z=(1 / 4) \eta^{2}, w=e^{z / 2} h, w=w(z)$. As a result, the general solution of Eq. (12) is

$$
w=C_{1} \Phi\left(\frac{1}{2}(1-k), \frac{1}{2} ; z\right)+C_{2} z^{1 / 2} \Phi\left(1-\frac{k}{2}, \frac{3}{2} ; z\right)
$$


where $C_{1}$ and $C_{2}$ are arbitrary constants, and $\Phi(a, b ; x)$ represents Kummer's confluent hypergeometric function. Working backward from this point, we arrive at the function

$$
f(\eta)=\frac{\eta\left[\begin{array}{l}
\Phi\left(\frac{1}{2}(1-k), \frac{1}{2} ; \frac{\eta^{2}}{4}\right) \\
+C \frac{\eta}{2} \Phi\left(1-\frac{k}{2}, \frac{3}{2} ; \frac{\eta^{2}}{4}\right)
\end{array}\right]+\left[\begin{array}{l}
(k-1) \eta \Phi\left(\frac{3-k}{2}, \frac{3}{2} ; \frac{\eta^{2}}{4}\right)-C \Phi\left(1-\frac{k}{2}, \frac{3}{2} ; \frac{\eta^{2}}{4}\right) \\
+C(k-2) \frac{\eta^{2}}{6} \Phi\left(2-\frac{k}{2}, \frac{5}{2} ; \frac{\eta^{2}}{4}\right)
\end{array}\right]}{\Phi\left(\frac{1}{2}(1-k), \frac{1}{2} ; \frac{\eta^{2}}{4}\right)+C \frac{\eta}{2} \Phi\left(1-\frac{k}{2}, \frac{3}{2} ; \frac{\eta^{2}}{4}\right)}
$$

and thus end up with a large family of the similarity solutions

$u(x, t)=\frac{\left\{\frac{(x+t)}{\sqrt{t}}\left[\begin{array}{l}\Phi\left(\frac{1}{2}(1-k), \frac{1}{2} ; \frac{(x+t)^{2}}{4 t}\right) \\ +C \frac{(x+t)}{2 \sqrt{t}} \Phi\left(1-\frac{k}{2}, \frac{3}{2} ; \frac{(x+t)^{2}}{4 t}\right)\end{array}\right]+\left[\begin{array}{l}(k-1) \frac{(x+t)}{\sqrt{t}} \Phi\left(\frac{3-k}{2}, \frac{3}{2} ; \frac{(x+t)^{2}}{4 t}\right) \\ -C \Phi\left(1-\frac{k}{2}, \frac{3}{2} ; \frac{(x+t)^{2}}{4 t}\right) \\ +C(k-2) \frac{(x+t)^{2}}{6 t} \Phi\left(2-\frac{k}{2}, \frac{5}{2} ; \frac{(x+t)^{2}}{4 t}\right)\end{array}\right]\right\}}{\sqrt{t} \Phi\left(\frac{1}{2}(1-k), \frac{1}{2} ; \frac{(x+t)^{2}}{4 t}\right)+C \frac{(x+t)}{2} \Phi\left(1-\frac{k}{2}, \frac{3}{2} ; \frac{(x+t)^{2}}{4 t}\right)}-1$

of Eq. (2), where $k \neq 0,1$ and $C$ is an arbitrary constant.

Case (5): $X_{5}=-t x \frac{\partial}{\partial x}-t^{2} \frac{\partial}{\partial t}+(t u-x) \frac{\partial}{\partial u}$.

From the characteristic system $\frac{\mathrm{d} x}{-t x}=\frac{\mathrm{d} t}{-t^{2}}=\frac{\mathrm{d} u}{t u-x}$, the invariants are obtained as $\eta=x / t, u=(1 / t) f(\eta)+(x / t)$ and thus Eq. (2) leads to reduced equation $f^{\prime \prime}=f^{\prime} f$ which creates shock soliton solutions as in Case (2).

The procedure described so far can be applied to any linear combination of the infinitesimal operators with constant coefficients. We include two more cases since they lead us to physically interesting invariant solutions of Eq. (2).

Case (6): $X_{1}+X_{2}+X_{3}=(t+1) \frac{\partial}{\partial x}+\frac{\partial}{\partial t}+\frac{\partial}{\partial u}$.

The corresponding one-parameter group is $\bar{x}=x+(t+1) \varepsilon, \bar{t}=t+\varepsilon$, $\bar{u}=u+\varepsilon$. Similarity variable and similarity transformation, respectively, are $\eta=x-t-t^{2} / 2$ and $u=t+f(\eta)$ obtained by solving the characteristic system $\frac{\mathrm{d} x}{t+1}=\frac{\mathrm{d} t}{1}=\frac{\mathrm{d} u}{1}$. The reduced equation is

$$
f^{\prime \prime}-f f^{\prime}+f^{\prime}-1=0
$$

and Eq. (14) can be written as

$$
f^{\prime}-\frac{1}{2} f^{2}+f-\eta=k
$$

which is a Riccati equation. Applying the transformation $f=-2 g^{\prime} / g$, $g=g(\eta)$, Eq. (15) leads to

$$
g^{\prime \prime}+g^{\prime}+\frac{1}{2}(\eta+k) g=0
$$

and again by using the transformation 


$$
g=e^{-\eta / 2} h(z), \quad z=\frac{1}{\sqrt[3]{2}} \eta+\left(\frac{k}{2}-\frac{1}{4}\right) / \frac{1}{\sqrt[3]{4}}
$$

Eq. (16) gives the equation

$$
h^{\prime \prime}+z h=0
$$

which is in normal form. Equation (17) has a particular solution

$$
h^{\prime \prime}=\sqrt{z} J_{1 / 3}\left(\frac{2}{3} z^{3 / 2}\right),
$$

where $J_{p}$ denotes a Bessel function. Working backward, we conclude that

$$
u(x, t)=1+t-\sqrt{2\left(x-t-\frac{t^{2}}{2}+k-\frac{1}{2}\right)} \frac{J_{-2 / 3}\left(\frac{\sqrt{2}}{3}\left(x-t-\frac{t^{2}}{2}+k-\frac{1}{2}\right)^{3 / 2}\right)}{J_{1 / 3}\left(\frac{\sqrt{2}}{3}\left(x-t-\frac{t^{2}}{2}+k-\frac{1}{2}\right)^{3 / 2}\right)}
$$

is a large family of invariant solutions of Eq. (2) in terms of the Bessel functions, where $k$ is an arbitrary constant.

We now find the one-parameter $(\varepsilon)$ family of solutions $u=\Phi(x, t, \varepsilon)$ of Eq. (2), obtained from any solution $u=\Theta(x, t)$ that is not in the form of (18), as

$$
u=\Phi(x, t, \varepsilon)=\Theta(x-(t-\varepsilon+1) \varepsilon, t-\varepsilon)+\varepsilon,
$$

resulting from the invariance of the Eq. (2) under the symmetry group $X_{1}+$ $X_{2}+X_{3}$.

Case (7): $X_{3}-X_{5}=(t+x t) \frac{\partial}{\partial x}+t^{2} \frac{\partial}{\partial t}+(1-t u+x) \frac{\partial}{\partial u}$.

The corresponding one-parameter group is $\bar{x}=\frac{x+\varepsilon t}{1-\varepsilon t}, \bar{t}=\frac{1}{1-\varepsilon t}, \bar{u}=u+(1-$ $t u+x) \varepsilon$. Similarity variable and similarity transformation, respectively, are $\eta=(x+1) / t$ and $u=\frac{1}{t} f(\eta)+\frac{x+1}{t}$ obtained by solving the characteristic system $\frac{\mathrm{d} x}{(1+x) t}=\frac{\mathrm{d} t}{t^{2}}=\frac{\mathrm{d} u}{1-t u+x}$. The reduced equation is $f^{\prime \prime}=f^{\prime} f$ and can be written as $f^{\prime}=\frac{1}{2} f^{2}+k$. Letting $f=-2 g^{\prime} / g, g=g(\eta)$ in the last equation leads to $g^{\prime \prime}+\frac{k}{2} g=0$, which is a second-order homogeneous constant coefficient linear equation. Finding the solution by the characteristics method and working backward, we get a large family of shock (kink soliton) solutions of Eq. (2) as

$$
\begin{aligned}
& u(x, t)=\frac{x+1}{t}+\frac{1}{t} \sqrt{2 k} \tan \left[\sqrt{\frac{k}{2}}\left(\frac{x+1}{t}-C\right)\right], \quad k>0, \\
& u(x, t)=\frac{x+1}{t}-\frac{1}{t} \sqrt{-2 k} \tanh \left[\sqrt{\frac{-k}{2}}\left(\frac{x+1}{t}-C\right)\right], \quad k<0,
\end{aligned}
$$

where $k$ is an arbitrary constant. 
We now find the one-parameter $(\varepsilon)$ family of solutions $u=\Phi(x, t, \varepsilon)$ of Eq. (2), obtained from any solution $u=\Theta(x, t)$ that is not in the form of (19a), (19b), as $u=\Phi(x, t, \varepsilon)=(1 /(1+\varepsilon t))[\Theta((z-\varepsilon t) /(1+\varepsilon t), t /(1+$ $\varepsilon t)+\varepsilon x]$, resulting from the invariance of Eq. (2) under the symmetry group $X_{3}-X_{5}$.

\section{Concluding remarks and discussions}

In this paper, the similarity solutions of Burgers' equation have been reached by using symmetry reductions of the Lie groups. Using the characteristic equations, new independent similarity variables and dependent similarity variables have been found. Then the reduced forms of the original Burgers' equation have been examined for distinct cases. Thus, similarity solutions of Burgers' equation have been obtained as a rich variety of exact analytic solutions. The results obtained in this paper are of general physics interest and should be readily verified, because the finding of new mathematical results might have significant impact on future research for physical sciences.

However, the symmetry groups of Burgers' equation considered so far in this paper have been local transformation groups acting "geometrically" on the space of independent and dependent variables. This fact, obviously, confines the determination of symmetry reductions of Lie groups. However, it is recognized that one can extensively extend the application of symmetry group methods by including derivatives of relevant dependent variables in their infinitesimal generators. This expansion, nowadays, is called "generalized symmetries" or "Noether transformations" [35]. In practice, the computation of all generalized symmetries of a given order is naturally feasible, and essentially the same as using classical symmetry reduction. One can calculate higher order generalized symmetries, but the computations are much more involved.

For example, Olver [35] computed all third order generalized symmetries of potential Burgers' equation, $v=v_{x x}+v_{x}^{2}$, where some of characteristic equations are affine on $u_{x x x}$ and listed all third order generalized symmetry of potential Burgers' equation (see, $c f$. Eq. (5.13) in Ref. [35]), but he did not study the integrability of the characteristic equations with third order derivatives, i.e., $u_{x x x}$. Because of these characteristics, computations grow rapidly more and more involved. However, the third order generalized symmetries of potential Burgers' equation imply implicitly that Burgers' equation shows dispersion and determination of the effect of dispersions on the solution(s) of Burgers' equation (2) cannot be determined even with straight application of generalized symmetries. The method of generalized symmetries suffers the disadvantage that the order of derivatives on which the coefficients of 
the symmetry dependence must be specified beforehand. This disadvantage can be overcome by introducing the methods such as Recursion Operators method, Master Symmetries or/and Formal Symmetries.

Returning to potential Burgers' equation, $=v_{x x}+v_{x}^{2}$, Olver [35] shows that the configuration of the resulting characteristics (see, $c f$. Eq. (5.13) in Ref. [35]) suggest that Burgers' equation has two recursion operators. The time derivative of the first recursion operator $R_{1}$ on solution to Burgers' equation is the multiplication operator $\left(R_{1}\right)_{t}=v_{x t}=v_{x x x}+2 v_{x} v_{x x}$. This multiplication operator coincides with the differential of potential Burgers' equation with respect to $x$. If we set $\frac{\delta v}{\delta x}=u$ so that $v$ can be taken as potential function, then resulting differential equation, after some manipulation, coincides with Burgers' equation. Thus, theoretically, there is an infinitive number of hierarchy of symmetries, with the characteristics mentioned.

On the other hand, recognizing that the Burgers' equation is the lowestorder approximation for the one-dimensional propagation of weak shock waves in a fluid, Kudryashov and Sinelshchikov [69] recently derived the equation for long weakly non-linear waves in the liquid with gas bubbles

$$
u_{t}+\alpha u u_{x}+\beta u_{x x x}-\mu u_{x x}-v\left(u u_{x}\right)_{x}+\gamma u_{x t}=0 .
$$

The reason of examining Eq. (20), at this stage, is not to study its properties but to take it as a representative of a class of Burgers' equations. In our case, Eq. (20) represents a toy model, making the problem tractable where the dispersion relation is not known explicitly, but the behaviour given by Eq. (20) can be established.

Consequently, if we consider the high-order terms with respect to small parameter, $\varepsilon$, Eq. (20) may be written as

$$
u_{t}+\alpha u u_{x}-\mu u_{x x}=\varepsilon\left(v\left(u u_{x}\right)_{x}-\beta u_{x x x}-\mu u_{x t}\right)
$$

which is the generalization of Burgers' equation for long weakly non-linear waves in the liquid with gas bubbles [70]. Differentiating Eq. (21) with respect to $x$, and substituting the result into Eq. (21), up to the first order in $\varepsilon$ it reads

$$
u_{t}+\alpha u u_{x}-\mu u_{x x}=\varepsilon\left((v+\mu \alpha)\left(u u_{x}\right)_{x}-\left(\beta+\mu^{2}\right) u_{x x x}\right) .
$$

Equation (22) is an evolution equation. By using near-identity transformations [71], a family of asymptotically equivalent equations for the representation of non-linear waves in liquid with gas bubbles is obtained. This equation can be given by

$$
\begin{aligned}
u_{t}+\alpha u u_{x}-\mu u_{x x}= & \varepsilon\left(\left(2 \mu \alpha_{2}+\mu \alpha+v\right) u u_{x}+\left(2 \mu \alpha_{1}+\mu \alpha+v\right) u_{x}^{2}\right. \\
& \left.-\frac{\alpha\left(\alpha_{2}+2 \alpha_{1}\right)}{2} u^{2} u_{x}-\left(\beta+\mu^{2}\right) u_{x x x}\right)
\end{aligned}
$$


where $t$ is the non-dimensional time, $x$ is the non-dimensional Cartesian co-ordinate, $u$ is the non-dimensional perturbation of gas-liquid mixture density, $\alpha, \beta, v, \mu$ are non-dimensional physical parameters, $\alpha_{1}$ and $\alpha_{2}$ are arbitrary parameters introduced by the near-identity transformation $[70,71]$. This equation is the sum of Burgers' equation and first higher order correction terms which will provide a link between the equations of the Burgers hierarchy and the evolution of a physical quantity. Equation (23) is integrable under certain conditions on parameters $\alpha_{1}, \alpha_{2}$ and $v$.

However, Kudryashov and Sinelshchikov [72] transformed Eq. (23) to the canonical form

$$
u_{t}-3\left(u u_{x}\right)_{x}+3 \alpha u^{2} u_{x}+u_{x x x}=0 .
$$

This equation is the second member of the Burgers hierarchy the so-called Sharma-Tasso-Olver (STO) equation [73-75]. Equation (24) is also interpreted as a perturbed Korteweg-de Vries equation given that some of terms of these equations are the same.

For instance, Kudryashov and Sinelshchikov [72] studied symmetries and symmetry reductions of Eq. (24) and noted that, in their case, the classical Lie method cannot imply the integrable case of Eq. (24). As an alternative, Kudryashov and Sinelshchikov [72] studied the classical symmetries for the potential form of this equation by setting $u=v_{x}$ in Eq. (24). For $\alpha=1$, equation (24) admits the infinitesimal generator $X_{\infty}=e^{v} \phi \partial_{v}$, where $\phi$ is a solution of third order linear dispersive waves

$$
\phi_{t}+\phi_{x x x}=0 .
$$

It is known that the third order derivative models dispersion where waves of different frequencies move at different speed as in the inviscid and viscous Burgers' equation possessing the same non-linearity.

They [72] implied that, since equation (25) is first order in $t$, one expects that its solutions are uniquely prescribed by their initial values, say, the fundamental solution of equation (25) corresponding to concentrated initial disturbance, $\phi(0, x)=\delta(x)$ can be given in terms of the Airy function

$$
\phi(t, x)=\frac{1}{\sqrt[3]{3 t}} \operatorname{Ai}\left(\frac{x}{\sqrt[3]{3 t}}\right) .
$$

Consequently, a solution to Burgers' equation with higher order correction terms (Eq. (21)) comes from a positive solution to the third order linear dispersive wave equation via the infinitesimal generator $X_{\infty}=e^{v} \phi \partial_{v}$, where $\phi$ is the solution given in (26). 
Beside this, Kudryashov and Sinelshchikov [72] considered the self-similar reduction of Eq. (24) using the variables

$$
u(x, t)=C_{1} t^{-\frac{1}{3}} f(\eta), \quad \eta=C_{2} x t^{-\frac{1}{3}} .
$$

For $C_{1}=-C_{2}=-(3)^{-\frac{1}{3}}$, Eq. (24) gives

$$
(\eta f)_{\eta}=3\left(f f_{\eta}\right)_{\eta}+3 \alpha f^{2} f_{\eta}+f_{\eta \eta \eta} .
$$

Integrating (27) with respect to $\eta$, one gets

$$
\eta f=3 f f_{\eta}+\alpha f^{3}+f_{\eta \eta}+C_{3},
$$

where $C_{3}$ is an integrating constant. Consulting Zaitsev and Polyanin [68], the general solution of Eq. (28) can only be attained in the case of $\alpha=1$. Yet again, at the arbitrary value of $\alpha$, Eq. (28) admits particular solution which is expressed in terms of Airy functions. In the case of $\alpha=0$, it is not possible to find the similarity solution of Eq. (24). On the other hand, one can obtain stationary solution of Eq. (24) at $\alpha=0$, that is, the solution is expressed in terms of Airy function. It is worth underlining that using the Cole-Hopf transformation, it is possible to obtain more general similarity solution of Eq. (24) at $\alpha=1$. This solution may be given in the form of [72]

$$
f=\frac{\Psi_{\eta}}{\Psi}, \quad \Psi=\int\left(C_{4} \operatorname{Ai}(\eta)+\operatorname{Bi}(\eta) \mathrm{d} \eta+C_{5},\right.
$$

where $C_{4}$ and $C_{5}$ are arbitrary constants.

Again, Kudryashov and Sinelshchikov [72] considered the theory of nonclassical symmetries [76-79] for reduction of Eq. (24), and they validated that the non-classical infinitesimal $\eta^{2}$ ( $c f$. Table 1 in Ref. [72]) corresponds to the exact solution of Eq. (24) that is expressed in terms of Airy functions.

So, as to remark, we can state that there are links between the generalization of Burgers' equation (21) and, consequently, equations (22)-(24) and basic Burgers' equation (2). Then, the possibility of solving many differential equations which arise in practical problems can often be traced to some geometrical or other symmetry property of the problem. It is apparent that the application of higher order terms in the state equation allows us to obtain a more exact solution of non-linear equations with the connection to the Burgers hierarchy. 
Now, let us consider Burgers' equation with an imaginary "viscosity" coefficient $v=i \epsilon$ given by

$$
\frac{\mathrm{d} \Psi}{\mathrm{d} t}=\Psi \frac{\mathrm{d} \Psi}{\mathrm{d} x}=i \epsilon \frac{\mathrm{d} \Psi^{2}}{\mathrm{~d} x^{2}}
$$

first introduced by Dobrokhotov et al. [80]. This equation is possibly the simplest model of a non-linear dispersive equation. Nevertheless, the model is non-linearly ill posed at least for certain complex values of $v$, since singularities can occur in finite time. However, Senouf et al. [81] considered that this equation is an motivating mathematical model for dispersive (imaginary $v$ ) or mixed dissipative-dispersive systems (complex $v$ ). In the analysis that follows, Senouf et al. [81], beside other cases (please refer to Ref. [81]), studied uniform asymptotic expansion of Eq. (30) as $v \rightarrow 0$ for $t>t_{*}$ across the caustic $x=x_{s}(t)$ in terms of the Pearcey integral which is obtained by following the notation of Kaminski [82]. Furthermore, this expansion is applicable outside the intervals centred about $x=\mp x_{s}(t)$ and these are the second order branch points of the inviscid solution [82]. But again, the region of interest is the neighbourhood of caustic. Hence, the uniform asymptotic expansion as $v \rightarrow 0^{+}$of $\Psi_{i \epsilon}\left(x=\mp x_{s}(t)-\delta_{\mp}(\tau ; t), t\right)$ in the neighbourhood of caustic $x=\mp x_{s}(t)$ is introduced in terms of the Airy functions ( $c f$. Property 5.3 in Ref. [81]). This uniform asymptotic expansion as $v \rightarrow 0^{+}$is, again, the dispersive solution in the neighbourhood of the caustic for Burgers' equation (30). Note finally that this work demonstrates the dispersive dynamics of the equation and $v=i \epsilon(\epsilon>0)$ is a purely dispersive coefficient.

For the arguments stated above, Burgers' equation grants approximations to more complex physical systems and can be taken as conservation laws and integrable system. Hence, these physical systems are very often obtained with the help of some asymptotic approach. On the other hand, taking into account higher order terms in the asymptotic approach (in the state equation), one can obtain generalization of Burgers' equation which confirm the presence of higher order contributions to the solutions. These solutions present a wealth of information on the original physical systems which are not accessible, for example as in our case, by the symmetry reductions of the Lie groups.

The above discussion reveals that certain integrable non-linear evolution equations can be obtained by limiting process involving rescaling and/or an asymptotic expansion from very large classes of non-linear equations. This is obtained via the change of dependent variable which supplies a suitable technique that all integrable equations are somehow related to each other and are the members of the hierarchy. This, of course, provides various possibilities to uncover non-trivial relations among integrable equations as in Burgers' hierarchy conferred above. Our debate here, of course, has focused 
only on the demonstration of explicit examples rather than a coherent treatment. Nonetheless, we maintain that the perturbed equation shares some of desirable properties of the unperturbed equation and the ability to generate perturbative expansions for the solutions depends on what type solutions we seek. Our inspiration has been the fact that unperturbed equations can be extended to the perturbed ones in an asymptotic sense as we illustrated above.

We would like to thank anonymous referees for their useful and valuable suggestions.

\section{REFERENCES}

[1] J.D. Logan, An Introduction to Nonlinear Partial Differential Equations, $2^{\text {nd }}$ Ed., John Wiley and Sons, 2008.

[2] J.M. Burgers, A Mathematical Model Illustrating the Theory of Turbulence, in: Advances in Applied Mechanics, vol. I, Academic Press, New York 1948, pp. 171-199.

[3] J.M. Burgers, The Nonlinear Diffusion Equation, Reiedl, Dordtrecht 1974.

[4] H. Bateman, Some Recent Researchers on the Motion of Fluids, Mon. Wea. Rev. 43, 163 (1915).

[5] L. Debnath, Nonlinear Partial Differential Equations for Scientists and Engineers, $3^{\text {rd }}$ Ed., Birkhauser, New York 2012.

[6] W.F. Ames, Nonlinear Partial Differential Equations in Engineering, Academic Press, New York 1972.

[7] E. Hopf, The Partial Differential Equation, Commun. Pure Appl. Math. 3, 201 (1950).

[8] J.D. Cole, On a Quasilinear Parabolic Equation Occurring in Aerodynamics, Q. Appl. Math. 9, 225 (1951).

[9] M.J. Lighthill, Viscosity Effects in Sound Waves of Finite Amplitude, in: Surveys in Mechanics, eds. G.K. Batchelor, R.M. Davies, Cambridge University Press, Cambridge 1956, pp. 250-351.

[10] D.T. Blackstock, Thermoviscous Attenuation of Plane, Periodic Finite Amplitude Sound Waves, J. Acoust. Soc. Am. 36, 534 (1964).

[11] R.A. Walsh, Initial-value Problems Associated with $u t(x, t)=\delta \operatorname{uxx}(x, t)-u(x, t) u x(x, t)$, J. Math. Anal. Appl. 26, 235 (1969).

[12] E.R. Benton, G.W. Platzman, Q. Appl. Math. 30, 195 (1972).

[13] D.G. Crighton, J.F. Scott, Philos. Trans. R. Soc. Lond. A 292, 101 (1979).

[14] D.F. Parker, Proc. R. Soc. London A 369, 409 (1980).

[15] E.Y. Rodin, J. Math. Anal. Appl. 30, 401 (1970).

[16] D.A. Larson, SIAM J. Appl. Math. 34, 93 (1978). 
[17] R.W. Lardner, J.C. Arya, Acta Mech. 37, 179 (1980).

[18] K.M. Case, C.S. Chiu, Phys. Fluids 12, 1799 (1969).

[19] J.D. Murray, SIAM J. Appl. Math. 19, 273 (1970).

[20] J.D. Murray, J. Fluid Mech. 44, 315 (1970).

[21] J.D. Murray, J. Fluid Mech. 59, 263 (1973).

[22] M. Rosenblatt, Phys. Fluids 9, 1247 (1966).

[23] P. Penel, C.M. Brauner, J. Math. Anal. Appl. 45, 654 (1974).

[24] D.G. Crighton, Annu. Rev. Fluid Mech. 11, 11 (1979).

[25] H.O. Kriess, J. Lorenz, Initial-Boundary Value Problems and the Navier-Stokes Equations, Academic Press, New York 1989.

[26] J. Kevorkian, Partial Differential Equations: Analytical Solution Techniques, $2^{\text {nd }}$ Ed., Springer-Verlag, New York 2000.

[27] A.M. Wazwaz, Appl. Math. Comput. 190, 1198 (2007).

[28] A.A. Soliman, Physica A 361, 394 (2006).

[29] A. Ebaid, Phys. Lett. A 365, 213 (2007).

[30] G. Adomian, Solving Frontier Problems of Physics: The Decomposition Method, Kluwer, Boston 1994.

[31] A.M. Wazwaz, Partial Differential Equations and Solitary Waves Theory, Springer, New York 2010.

[32] M.J. Ablowitz, H. Segur, Solitons and Inverse Scattering Transform, SIAM, Philadelphia 1981.

[33] R. Hirota, Direct Methods in Soliton Theory, in: Solitons, Springer-Verlag, 1980.

[34] M.J. Ablowitz, A. Ramani, H. Segur J. Math. Phys. 21, 715 (1980).

[35] P.J. Olver, Applications of Lie Groups to Differential Equations, Springer-Verlag, New York 1986.

[36] W.F. Ames, M.C. Nucci, J. Eng. Math. 20, 181 (1986).

[37] W. Chester, Journal of Institute of Mathematics and Its Applications 19, 343 (1977).

[38] E.Y.A. Rodin, Q. Appl. Math. 27, 541 (1974).

[39] M. Tajiri, S. Kawamoto, K. Thushima, Math. Japonica 28, 125 (1983).

[40] S. Hood, J. Math. Phys. 36, 1971 (1995).

[41] P.A. Clarkson, M.D. Kruskal, J. Math. Phys. 30, 2201 (1989).

[42] E.L. Miller, Predictor-corrector Studies of Burger's Model of Turbulent Flow, M.S. Thesis, University of Delaware, Newark, Delaware, 1966.

[43] J. Caldwell, P. Wanless, Appl. Math. Modelling 5, 189 (1981).

[44] J. Caldwell, P. Smith P, Appl. Math. Modelling 6, 381 (1982).

[45] J. Caldwell, P. Wanless, Appl. Math. Modelling 11, 211 (1987).

[46] R.C. Mittal, P. Singhal, Commun. Numer. Meth. Eng. 9, 397 (1993). 
[47] Y.C. Hon, X.Z. Mao, Appl. Math. Comput. 95, 37 (1998).

[48] T. Öziş, A. Özdeş, J. Comput. Appl. Math. 71, 163 (1996).

[49] S. Kutluay, A.R. Bahadir, A. Özdeş, J. Comput. Appl. Math. 103, 251 (1999).

[50] M.B. Abd-el-Malek, S.M.A. El-Mansi, J. Comput. Appl. Math. 115, 1 (2000).

[51] T. Öziş, E.N. Aksan, A. Özdeş, Appl. Math. Comput. 139, 417 (2003).

[52] S. Kutluay, A. Esen, Appl. Math. Comput. 156, 295 (2004).

[53] S. Abbasbandy, M.T. Darvishi, Appl. Math. Comput. 163, 1265 (2005).

[54] T. Öziş, A. Esen, S. Kutluay, Appl. Math. Comput. 165, 237 (2005).

[55] M. Gülsu, T. Öziş, Appl. Math. Comput. 171, 1192 (2005).

[56] T. Öziş, Y. Aslan, Appl. Math. Comput. 163, 131 (2005).

[57] E.N. Aksan, A. Özdeş, T. Öziş, Appl. Math. Comput. 176, 270 (2006).

[58] T. Öziş, U. Erdoğan, Int. J. Numer. Meth. Eng. 79, 696 (2009).

[59] M. Seydaoğlu, U. Erdoğan, T. Öziş, J. Comput. Appl. Math. 291, 410 (2016).

[60] G.W. Bluman, S.C. Anco, Similarity and Integration Methods for Differential Equations, Springer-Verlag, New York 2002.

[61] N.H. Ibragimov, CRC Handbook of Lie Group Analysis of Differential Equations, Vol. 1, CRC Press, Florida, 1994.

[62] N.H. Ibragimov, Transformation Groups and Lie Algebras, Higher Education Press Limited Company, Beijing 2013.

[63] L.V. Ovsiannikov, Group Analysis of Differential Equations, Academic Press, New York 1982.

[64] P.G.L. Leach, K.S. Govinder, Symmetry in Mechanics. Modern Group Analysis, V (Johannesburg, 1994), Lie Groups Appl. 1, 146 (1994).

[65] M. Felsand, P.J. Olver, Moving Frames and Coframes. In Algebraic Methods in Physics, Montreal, QC, 1997, CRMSer. Math. Phys., 2001, pp. 47-64, Springer-Verlag, New York.

[66] P.J. Olver, An Introduction to Moving Frames. In Geometry, Integrability and Quantization, papers from the $5^{\mathrm{th}}$ International Conference held in Varna, June 5-12, 2003, I.M. Mladenov, A.C. Hirshfeld, eds., pp. 67-80, Softex, Sofia 2004.

[67] P.J. Olver, J. Pohjanpelto, Canad. J. Math. 60, 1336 (2008).

[68] V.F. Zaitsev, A.D. Polyanin, Handbook of Exact Solutions for Ordinary Differential Equations, CRC Press, Boca Raton 2002.

[69] N.A. Kudryashov, D.I. Sinelshchikov, Wave Motion 50, 351 (2013).

[70] N.A. Kudryashov, D.I. Sinelshchikov, Int. J. Nonlin. Mech. 63, 31 (2014).

[71] A. Veksler, Y. Zarmi, Physica D 211, 57 (2005). 
[72] N.A. Kudryashov, D.I. Sinelshchikov, Phys. Lett. A 379, 798 (2015) [arXiv:1608.00811 [nlin.SI]].

[73] H. Tasso, J. Phys. A: Math. Gen. 29, 7779 (1996).

[74] P.J. Olver, J. Math. Phys. 18, 1212 (1977).

[75] N.A. Kudryashov, D.I. Sinelshchikov, Appl. Math. Comput. 215, 1293 (2009).

[76] G.W. Bluman, J.D. Cole, J. Math. Mech. 18, 1025 (1969).

[77] D. Levi, P. Winternitz, J. Phys. A: Math. Gen. 22, 2915 (1989).

[78] O.A. Pocheketa, R.O. Popovych, J. Math. Anal. Appl. 398, 270 (2013).

[79] M.S. Bruzon, M.L. Gandarias, M. Torrisi, R. Tracinà, J. Math. Phys. 50, 103504 (2009).

[80] S.Y. Dobrokhotov, V.P. Maslov, V.B. Tsvetkov, Mate. Zametki 51, 143 (1992).

[81] D. Senouf, R. Caflisch, N. Ercolani, Nonlinearity 9, 1671 (1996).

[82] D. Senouf, SIAM J. Math. Anal. 28, 1457 (1997). 